\title{
The challenges of implementing evidence-based practices in pediatric neurosurgery
}

\author{
Shenandoah Robinson, MD \\ Division of Pediatric Neurosurgery, Johns Hopkins University School of Medicine, Baltimore, Maryland
}

$\mathrm{D}$ R. Kraemer and colleagues are to be congratulated for taking on the challenge of deciphering the shunt practice patterns of seasoned North American pediatric neurosurgeons. Shunt management for hydrocephalus in children remains a pressing clinical problem. Despite advances in shunt protocols and hardware, shunts to treat hydrocephalus remain troublesome devices, and patients continue to experience pain and morbidity. Families must deal with the stress of caring for children with chronic, potentially life-threatening illnesses. The diagnosis of hydrocephalus can carry lifechanging medical, surgical, and behavioral consequences. And families must often cope with significant financial burdens. Hospital admissions and surgeries for shunt malfunction and infection consume enormous health care resources each year.

In the report of their survey-based study, entitled "Shunt-dependent hydrocephalus: management style among members of the American Society of Pediatric Neurosurgeons," the authors attempt to take an educated snapshot of practical clinical judgment based on the responses of experienced surgeons to commonly encountered clinical scenarios. ${ }^{7}$ The authors' goal was to evaluate differences in the evaluation and management of shuntdependent hydrocephalus by using a targeted questionnaire. Overall, 130 senior pediatric neurosurgeons participated as members of the American Society of Pediatric Neurosurgeons, with a response rate of $64 \%$. Multiplechoice questions were posed and free-text responses were solicited. The 4 main topics covered were the management of shunt malfunction, chronic shunt overdrainage, chronic headaches in shunt patients, and slit ventricle syndrome.

The first topic focused on identifying the preferred shunt valve for most pediatric patients and the most common cause of shunt malfunction. Consistent with welldesigned shunt valve trials that have shown that no shunt valve performs better than another, ${ }^{1}$ pediatric neurosur- geons continue to use a wide variety of valves. As primary shunt insertion and revision practices become more standardized through the use of protocols and best practices, we may be able to clarify whether certain valve designs perform better in specific populations. If it remains the case that no one valve design is superior to another, then the direct cost of valves and the resources to manage them, including additional imaging and personnel time required for reprogramming, should be weighed and optimized.

The section on shunt malfunction also asked neurosurgeons to identify the type of material that obstructs the holes in ventricular catheters. The majority of respondents named the choroid plexus, reflecting the findings of studies from the 1960s and 1970s. ${ }^{2,5}$ As the authors note, the most recent study on this topic implicates a reactive and/or inflammatory process with microglia and astrocytes, presumably in response to the foreign material of the catheter tubing itself. ${ }^{6}$ That study was published in $2016-2$ years after the survey for this report was performed. Even so, this example serves as a reminder that keeping abreast of new advances in basic and translational science relevant to pediatric neurosurgery remains an ongoing challenge for busy surgeons.

The second topic the authors addressed was chronic shunt overdrainage. They noted that understanding the true nature of overdrainage requires the use of more precise definitions. A lack of clear definitions for specific shunt-related problems remains a significant limitation in pediatric neurosurgery. Unlike many other fields of medicine that long ago adopted consensus definitions, progress in pediatric neurosurgery research is hampered by a lack of precise definitions of problems and clinical outcomes.

This survey also investigated the management of chronic headaches in shunt-dependent hydrocephalus. Patients with shunts report a high prevalence of chronic headaches: $29 \%$ of children and $42 \%-44 \%$ of adolescents and young 
adults. ${ }^{9}$ The authors found that most pediatric neurosurgeons use reassurance and/or referral to neurology or pain division-based headache clinics to manage chronic pain in shunt patients. As Kraemer and colleagues note, pain management strategies for typically developing non-shunt patients may not be effective for shunt-dependent children and young adults. Current concepts of chronic pain suggest that a person's perception of pain, in large part, is based on attention and brain network activity, especially the default mode network. ${ }^{8}$ There are few studies of the default mode network and pertinent related networks in adolescents and young adults born very preterm or with myelomeningoceles. ${ }^{3,4,10}$ But current results demonstrate that these patients have abnormal networks. ${ }^{3,4,10}$ Pediatric neurosurgeons need to advocate for more research about shunt-dependent hydrocephalus to understand the relevant pathophysiology and to develop interventions specific for patients with this condition. Currently, very few evidencebased recommendations are available to treat chronic headaches in patients with congenital brain abnormalities, including those with shunted hydrocephalus.

Finally, the authors addressed the management of slit ventricle syndrome. Again, information about management and outcomes is limited due to the lack of consensus on definitions. The lack of agreement about management strategies probably reflects the array of definitions of the problem and of successful, measurable outcomes.

While this study has the inherent limitations of a voluntary survey, the results serve as a call to action for more refined definitions of specific problems and outcomes that will allow rigorous and reproducible studies to be designed and implemented to change future shunt management. Pediatric neurosurgery is a small field, and the challenges are often overwhelming for practitioners with limited time and resources. Ideally, advances in neuroscience will lead to strategies to reduce the number of individuals dependent on CSF diversion with a shunt. In the meantime, as children with hydrocephalus survive longer, there is a pressing need to reduce the burden of shunt-dependent hydrocephalus for our patients, their families, and society.

https://thejns.org/doi/abs/10.3171/2017.3.PEDS17103

\section{References}

1. Baird LC, Mazzola CA, Auguste KI, Klimo P Jr: Pediatric hydrocephalus: systematic literature review and evidencebased guidelines. Part 5: effect of valve type on cerebrospinal fluid shunt efficacy. J Neurosurg Pediatr 14 (Suppl 1):35-43, 2014

2. Collins P, Hockley AD, Woollam D: Surface ultrastructure of tissues occluding ventricular catheters. J Neurosurg 48:609-613, 1978

3. Daamen M, Bauml JG, Scheef L, Sorg C, Busch B, Baumann $\mathrm{N}$, et al: Working memory in preterm-born adults: load-dependent compensatory activity of the posterior default mode network. Hum Brain Mapp 36:1121-1137, 2015

4. Dennis M, Cirino PT, Simic N, Juranek J, Taylor WP, Fletcher JM: White and gray matter relations to simple, choice, and cognitive reaction time in spina bifida. Brain Imaging Behav 10:238-251, 2016

5. Hakim S: observation on the physiopathology of the CSF pulse and prevention of ventricular catheter obstruction in valve shunts. Dev Med Child Neuro Suppl 20:42-48, 1969
6. Hanak BW, Ross EF, Harris CA, Browd SR, Shain W: Toward a better understanding of the cellular basis for cerebrospinal fluid shunt construction: report on the construction of a bank of explanted hydrocephalus devices. J Neurosurg Pediatr 18:212-223, 2016

7. Kraemer MR, Sandoval-Garcia C, Bragg T, Iskandar BJ: Shunt-dependent hydrocephalus: management style among members of the American Society of Pediatric Neurosurgeons. J Neurosurg Pediatr [epub ahead of print June 30, 2017. DOI: 10.3171/2017.2.PEDS16265]

8. Kucyi A, Davis KD: The dynamic pain connectome. Trends Neurosci 38:86-95, 2015

9. Rekate HL, Kranz DA: Headaches in patients with shunts. Semin Pediatr Neurol 16:27-30, 2009

10. White TP, Symington I, Castellanos NP, Brittain PJ, Froudist Walsh S, Nam KW, et al: Dysconnectivity of neurocognitive networks at rest in very-preterm born adults. Neuroimage Clin 14:352-365, 2014

\section{Disclosures}

The author reports no conflict of interest.

\section{Response}

\section{Bermans J. Iskandar, MD, and Mark R. Kraemer, MD}

\section{Department of Neurosurgery, University of Wisconsin Hospitals and Clinics, Madison, Wisconsin}

We thank Dr. Robinson and the editorial board of the Journal of Neurosurgery: Pediatrics for the thoughtful comments regarding our paper. The motivations for this survey are clear: First and foremost, hydrocephalus of different etiologies represents nearly half of a typical pediatric neurosurgical practice; ventricular shunts constitute our specialty's most life-saving intervention; and shunt-related morbidity arguably accounts for the majority of pediatric neurosurgical complications. Furthermore, according to the literature and the neurosurgical community's collective experience, no shunt valve manufactured in the past 60 years has improved upon the rate of shunt complications with John Holter's original design. Accordingly, we argue that it is difficult to conceive of a pediatric neurosurgical problem or research area of greater impact.

Why 60 years with little progress? Why do up to $40 \%$ of shunts fail within a year of implantation and most fail within a decade? It is not the lack of adequate technology, nor the absence of intelligent minds to apply to the problem, and certainly not a want of effort. The Hydrocephalus Clinical Research Network is a clear testament of how the field can be advanced when experts team up and devote their efforts to a common objective. Rather, the answer is universal. As Albert Einstein declared, "Any fool can know. The point is to understand." Our survey of the senior community of North American pediatric neurosurgeons attests to how poorly we understand the causes of shunt obstruction and slit ventricle syndrome and the high prevalence of debilitating headaches. Consequently, significant disparities exist in the management of these entities. The survey stands as both a humbling reminder of our current status and, as Dr. Robinson duly notes, a 
call to action. We should feel accountable for the lack of progress of the past decades and procure stewardship of the future because no other professional group has as much stake in, or involvement with, this patient population. Neurologists, pain specialists, and scientists as well as biomedical engineers and medical device companies are essential in our quest to improve the care of our young patients with hydrocephalus, but they are heavily dependent on our counsel and collaboration in both research planning and implementation.

Dr. Robinson, when she enumerates the medical, neurological, psychosocial, and financial burdens produced by this otherwise life-saving device, puts into words the thoughts of virtually all who care for patients with shunts. Notwithstanding, her most compelling reflection is that at the root of the challenge, there is "a lack of precise definitions of problems and clinical outcomes," leading to "very few evidence-based recommendations." The present survey answers fewer questions than it generates. Yet, we hope that it will help to motivate renewed enthusiasm for research and development and, in particular, renewed commitment to a better understanding of shunt technology and physiology and a shunt's long-term impact on the brain. 